
\title{
25 Research Square \\ Expression Profiling of Growth Specific Genes Associated Silk Productivity in Bombyx Mori
}

\section{Ashok Kumar K}

Central Silk Board, Ministry of Textile: Govt of India Srinagar, India

Premnath Dhanaraj ( $\sim$ prems.bioinfo@gmail.com )

Karunya Institute of Technology and Sciences https://orcid.org/0000-0002-1969-4917

Indiraleka Muthiah

Mepco schlenk Engineering College, Sivakasi ,India

Anbu M Gomez

Karunya Institute of Technology and Sciences ,Coimbatore

Satish Lakkakula

Ben-Gurion University of Negev Israel

\section{Research Article}

Keywords: Silkworm, EST, RFLP- (STS) molecular markers

Posted Date: May 5th, 2021

DOI: https://doi.org/10.21203/rs.3.rs-451324/v1

License: (c) (1) This work is licensed under a Creative Commons Attribution 4.0 International License. Read Full License 


\section{Abstract}

Silkworm Bombyx mori exhibits wide variability and development attributes. The genetics of yield expression, shown to be of polygenic nature, is poorly studied in silkworm, to identify markers associated with 5 selected yield traits, with two RFLP-derived sequence -tagged site (STS) primers on the genomic DNA of silkworm germplasm stocks of different origin and diverse yield potential. The analysis led to the identification of two markers showing significant association with the yield traits. The markers could classify the stocks according to yield potential, irrespective of their origin. The relevance of the STS primers is discussed in the context of Appling multiple regression models for identifying markers associated with yield expression and stability for molecular breeding work in B. mori for yield improvement.

\section{Introduction}

Sericulture is an important rural agro-industrial practice in India and all the major commercial silks are produced in the country [1]. Sericulture, the practice of breeding silkworms for the production of raw silk, has been underway for at least 5000 years in China, from where it was spread to Korea and Japan and later to India and the west. The prestigious silk fiber is produced by the monophagous insect, the silkworm Bombyx mori, a Lepidopteron; whose sole food is mulberry. Silkworm is a holometabolous insect, which completes its life cycles of four different metamorphosing phases i.e., egg, larva, pupa and adult $50-55$ days.

More than 4000 Bombyx mori strains are currently available throughout the world and these strains are maintained by continuous sibling mating. At Central Sericultural Germplasm Resources Centre (CSGRC), Hosur, Tamil Nadu, where it was undertaken around 450 strains of silkworm are maintained and conserved.

The gene pool available in the country can be broadly divided in to two groups, the low yielding stocks characterized by high adaptability to tropical conditions and the yielding stocks exhibiting regular diapause, but suffer from the low adaptability to the highly variable tropical agro-climatic conditions $[2,3]$ and are mainly developed from Japanese hybrids[4]. The proposed 'gene tagged breeding' to combine the best qualities of the temperate high yielding stocks with tropical hardy ones $[5,6]$.

The analyzed many cDNA libraries prepared from various tissues[7] and at different developmental stages that cover almost entire set of Bombyx genes, comprising 35,000 expressed sequence tags (EST) from $36 \mathrm{cDNA}$ libraries. Expressed sequence Tags or ESTS are small pieces of DNA sequences that are generated by sequencing either one or both ends of an expressed gene. Once generated, they are useful in cloning specific genes of interest and mapping of functional genes in various related organisms. Such markers are obtained by partial sequencing of random clones. ESTs are popularly used in full genome sequencing and mapping program underway for a number of organisms and for identifying active genes thus helping in identification of diagnostic markers. EST is very simple tool for genome analysis. ESTs 
provide researchers with a quick and inexpensive route for discovering new genes, for obtaining data on gene expression and regulation, and for constructing the genome maps. EST database was also used to develop simple sequence repeat (SSR) markers for various species.

To build a foundation for the complete genome analysis of the silkworm, Bombyx mori, EST database has been constructed [8] covering about $55 \%$ of all genes of silkworm. EST's (Expressed Sequence Tags) are small pieces of DNA sequence, usually 200-500 nucleotides long, that are generated by either one or both ends of a cDNA. ESTs are presently being used as markers for genome mapping and identification of expressed genes. ESTs libraries and databases have proven to be powerful tools for gene discovery, gene mapping, and for the analysis of quantitative traits. ESTs are generated by large-scale sequencing of randomly picked clones from cDNA libraries constructed from mRNA isolated at a particular development stage and/or tissue. Japanese scientists are actively involved in the genome analysis in the mulberry silkworm. It has a large collection of cDNA libraries prepared from various and different developmental stages to cover the entire set of silkworm's genes. It is also engaged in construction of BAC (Bacterial Artificial Chromosome) library and making BAC contigs based on DNA fingerprinting and EST markers anchored to linkage maps.

Vitellogenins, the precursors of major yolk proteins of oviparous animals, Vitellogenin of the silkworm, Bombyx mori, is a tetramer with molecular weight of $440 \mathrm{~K}$,composed of each two molecules of nonidentical subunits termed heavy chain and light chain [9].

The prothoracic gene of Bombyx mori is a polymorphic one. The gene for the prothoracicotropic hormone (Ptth) in Bombyx mori has been already cloned and characterized. Three alleles a,b,c were identified in the Ptth locus which encodes PTTH in the silkworm ,Bombyx mori. These three alleles can be easily diagnosed by using the PCR technique because their length of the 3rd and 4th introns varies depending on the genotype [10].

Silk fibroin is secreted into the lumen of the posterior silk gland. Silk fibroin is composed of one heavy chain $(\approx 350 \mathrm{KDa})$ and one light chain $(25 \mathrm{KDa})[11,12]$. The identified a new mRNA species in the posterior silk gland encoding a silk protein of $25.197 \mathrm{KDa}$ which was termed as $\mathrm{P}_{25}$. $\mathrm{P}_{25}$ protein is produced in equimolar concentrations to major Fibroin $[13,14]$.

Therefore, in the present study, organization of yolk protein gene and PTTH gene genetic diversity were analyzed and their variations associated with productive traits in silkworm germplasm

\section{Materials And Methods}

\section{Silkworm Races}

A total 105 Silkworm accessions (30 MV and 75BV accessions) were screened using growth gene (PTTH) and Yolk protein gene primers. Silkworm strains from different origin, parentage and donor were chosen 
from the silkworm stocks based on the yield diversity. Strains were maintained at Central Sericultural Germplasm Resources Centre, Hosur, Tamil Nadu. Data's were given in table 1 \& 2.

\section{Genomic DNA isolation}

Genomic DNA was isolated from all the selected strains by standard phenol chloroform method (Nagaraja and Nagaraju, 1995). Extraction buffer (100Mm tris HCL, pH 8.0, 50Mm Nacl, 50,50Mm EDTA and $1 \%$ SDS $)$ and proteinase $\mathrm{k}(100 \mu \mathrm{g} / \mathrm{ml})$ was added to the ground tissue and incubated at $37^{\circ} \mathrm{C}$. Then DNA was extracted with phenol-chloroform-isoamylalcohol. DNA was dissolved in TE (10Mm Tris-HCL, $1 \mathrm{Mm}$ EDTA, pH 8.0). The genomic DNA was quantified in $0.8 \%$ Agarose gels and diluted to a uniform concentration $(20 \mathrm{ng} / \mu \mathrm{L})$. PCR was carried out with two expressed sequence tag primers (EST) viz., PTTH and yolk protein gene and then PCR products were restricted with EcoRI restriction enzyme.

\section{Primer Designing}

The up and down gene-specific primers were designed for available gene sequence of yolk protein gene and PTTH gene using the software programme of primer3 [15]

\section{PCR condition and analysis of amplified product}

The amplification of genomic DNA was performed according to the amplification reaction was carried out in a $20 \mu \mathrm{L}$ reaction volume containing $20 \mathrm{ng}$ of template DNA, 10×PCR buffer (10Mm Tris- HCL, $\mathrm{pH}$ 8.3,1.5Mm Mgcl2 and 50MmKCL), $0.2 \mu \mathrm{M}$ primer, $100 \mu \mathrm{M}$ each of dATP, dCTP, dGTP and dTTP, and 3units of Taq DNA polymerase (genei) [16]. Amplification was performed in a MJ RESEARCH Peliteir thermal cycler (PTC 200 Controller). PCR schedule was $94^{\circ} \mathrm{C}$ for $2 \mathrm{~min}$, followed by $45 \mathrm{cycles}$ of $94^{\circ} \mathrm{C}$ for $30 \mathrm{sec}$, $55^{\circ} \mathrm{C}$ for $30 \mathrm{sec}$ and $72{ }^{\circ} \mathrm{C}$ for $2 \mathrm{~min}$ and final extension was at $72{ }^{\circ} \mathrm{C}$ for 10 minutes. The amplified products were resolved on $1.5 \%$ Agarose gels in TBE buffer (89Mm Tris, $89 \mathrm{Mm}$ boric acid and $2 \mathrm{Mm}$ EDTA) and electrophoresis was carried out with a constant voltage. gels were stained with ethidium bromide $(0.5 \mathrm{ug} / \mathrm{ml})$ and analysis of restriction banding pattern were carried out using $1.5 \%$ Agarose gel, and photographed through gel documentation system. The presence of an amplified product was identified as 1 and the absence was designated as 0

\section{Statistical Data Analysis}

The SPSS/PC-11.5 (M.J.Norusis,SPSS Inc.,Chicago) package $[17,18]$ was used for various statistical analyses. The PCR profiles were subjected to binary scoring ( 1 for presence and 0 for absence of DNA fragments).Clustering of 105 genotypes was done on genetic distance. Genetic similarity coefficients among twenty races were estimated from the binary data by Hierarchical cluster analysis using Ward method [19-21]. Two major groups are found in the cluster obtained.

\section{Results And Discussion}


A total 105 Silkworm accessions (30 MV and 75BV accessions) were screened using growth gene (PTTH) and Yolk protein gene primers. The DNA restriction patterns enzymes showed three band patterns which are designated $a s a, b$ and $c$. The presence and absence of these three types were entered in the ward's minimum variance test for all the $75 \mathrm{BV}$ and $30 \mathrm{MV}$ accessions. Three restriction banding pattern were observed among the screened silkworm genetic resources and their scoring revealed high, moderate and low fecundity and growth groups

The PCR product of PTTH and Yolk protein genes (Figs. 1 \& 2) after digestion with restriction enzymes showed three band patterns which are designated as a, b and c. The presence and absence of these three types were entered in the ward's minimum variance test for all the $75 \mathrm{BV}$ and $30 \mathrm{MV}$ accessions (Figs. 3 \& 4). Cluster groups were identified into high, moderate and low productive groups. The presence of allele / band a \& b were seen among high productive group and a and $\mathrm{c}$ in the moderate and low productive groups (Tables $3 \& 4$ ). Three restriction banding pattern were observed among the screened silkworm genetic resources and their scoring revealed high, moderate and low fecundity and growth groups aa, ab banding types were found in Bivoltine high yielding groups and ac, bc found in high yielding Multivoltine groups.

The prothoracic gene of Bombyx mori is a polymorphic one. The gene for the prothoracicotropic hormone in Bombyx mori has been already cloned and characterized Three alleles a,b,c were identified in the ptth locus which encodes PTTH in the silkworm ,Bombyx mori. These three alleles can be easily diagnosed by using the PCR technique because their length of the 3rd and 4th introns varies depending on the genotype $[22,23]$.

\section{Conclusion}

Express Sequence Tag site technique was employed as an important molecular tool to identify productive breeds among promising silkworm genetic resources. Identified productive races based on growth and yield specific genes can be used as high productive/robust breeds by the breeders for marker assisted selection breeding to evolve robust breed for field utilization and augmentation of silk productivity in the field.

\section{Declarations}

\section{Acknowledgement}

The authors express sincere thanks to the S. Sekar, Assistant Director (computer) and competent authority of Central Silk Board, Ministry of Textiles, Madivala, Bangalore, Karnataka and Karunya Institute of Technology and Sciences department of Biotechnology for providing lab facility.

\section{References}


1. Krishanswami S, Narasimhanna MN, Suryanaruyana SK, Kumararaj s (1973) Silkworm Rearing Bulletin 15/2 FAO Agricultural Services. United Nations Organizations, Rome, pp 53-90

2. Chatterjee SN, Mohandas TP (2003) Identification of ISSR markers associated with productivity traits in silkworm, Bombyx mori L. Genome 46:438-447

3. Chatterjee SN, Rao CGP. Chatterjee GK, Ashwath SK and A.K.Patnaik (1993) A correlation between yield and biochemical parameters in the mulberry silkworm, Bombyx mori L. Theor Appl Genet 87:385-391

4. Chatterjee SN, Datta RK (1992) Hierarchical clustering of 54 races and strains of the mulberry silkworm, Bombyx mori L: Significance of biochemical Parameters. Theor Appl Genet 85:394-402

5. Goldsmith MR, Wilkins AS (eds) (1991) Molecular model Systems of the Lepidoptera. Cambridge University Press, New York, pp 21-76

6. Gupta PK, Varshney RK, Sharma PC, Ramesh B (1999) Molecular markers and their applications in wheat breeding. Plant breeding 118(5):369-390

7. Xing L, Liu XM (2012) Characterization of Betula platyphylla gene transcripts associated with early development of male inflorescence. Molecular biology reports 39(2):929-935

8. Duan J, Li R, Cheng D, Fan W, Zha X, Cheng T, ... Xia Q (2010) SilkDB v2. 0: a platform for silkworm (Bombyx mori) genome biology. Nucleic acids research 38(suppl_1):D453-D456

9. Rapali P, Szenes Á, Radnai L, Bakos A, Pál G, Nyitray L (2011) DYNLL/LC8: a light chain subunit of the dynein motor complex and beyond. FEBS J 278(17):2980-2996

10. Gomes FE, Arantes TD, Fernandes JA, Ferreira LC, Romero H, Bosco SM, ... Theodoro RC (2018) Polymorphism in mitochondrial group I introns among Cryptococcus neoformans and Cryptococcus gattii genotypes and its association with drug susceptibility. Frontiers in microbiology 9:86

11. Wadbua P, Promdonkoy B, Maensiri S, Siri S (2010) Different properties of electrospun fibrous scaffolds of separated heavy-chain and light-chain fibroins of Bombyx mori. Int J Biol Macromol 46(5):493-501

12. Inoue S, Tanaka K, Arisaka F, Kimura S, Ohtomo K, Mizuno S (2000) Silk fibroin of Bombyx mori is secreted, assembling a high molecular mass elementary unit consisting of $\mathrm{H}$-chain, L-chain, and P25, with a 6: 6: 1 molar ratio. J Biol Chem 275(51):40517-40528

13. Mandal BB, Grinberg A, Gil ES, Panilaitis B, Kaplan DL (2012) High-strength silk protein scaffolds for bone repair. Proceedings of the National Academy of Sciences, 109(20), 7699-7704

14. Zhang YQ (2002) Applications of natural silk protein sericin in biomaterials. Biotechnology advances 20(2):91-100

15. Kasnauskiene J, Ciuladaite Z, Preiksaitiene E, Matulevičienè A, Alexandrou A, Koumbaris G, ... Kučinskas V (2012) A single gene deletion on 4q28. 3: PCDH18-a new candidate gene for intellectual disability? Eur J Med Genet 55(4):274-277

16. Ramakers C, Ruijter JM, Deprez RHL, Moorman AF (2003) Assumption-free analysis of quantitative real-time polymerase chain reaction (PCR) data. Neurosci Lett 339(1):62-66 
17. Frey F (2017) SPSS (Software). The International Encyclopedia of Communication Research Methods, 1-2

18. Gouda MA (2015) Common pitfalls in reporting the use of SPSS software. Medical Principles Practice 24(3):300

19. Saraçli S, Doğan N, Doğan i (2013) Comparison of hierarchical cluster analysis methods by cophenetic correlation. Journal of inequalities Applications 2013(1):1-8

20. Granato D, Santos JS, Escher GB, Ferreira BL, Maggio RM (2018) Use of principal component analysis (PCA) and hierarchical cluster analysis (HCA) for multivariate association between bioactive compounds and functional properties in foods: A critical perspective. Trends Food Sci Technol 72:83-90

21. Zolfaghari F, Khosravi H, Shahriyari A, Jabbari M, Abolhasani A (2019) Hierarchical cluster analysis to identify the homogeneous desertification management units. PloS one 14(12):e0226355

22. Gomes FE, Arantes TD, Fernandes JA, Ferreira LC, Romero H, Bosco SM, ... Theodoro RC (2018) Polymorphism in mitochondrial group I introns among Cryptococcus neoformans and Cryptococcus gattii genotypes and its association with drug susceptibility. Frontiers in microbiology 9:86

23. Sorkheh K, Shiran B, Gradziel TM, Epperson BK, Martínez-Gómez P, Asadi E (2007) Amplified fragment length polymorphism as a tool for molecular characterization of almond germplasm: genetic diversity among cultivated genotypes and related wild species of almond, and its relationships with agronomic traits. Euphytica 156(3):327-344

\section{Tables}

Table1.Allelic variations in PTTH \& yolk protein gene primers in Bivoltine accessions 


\begin{tabular}{|c|c|c|c|c|c|c|c|c|c|c|}
\hline ACC.NO & Races & $\begin{array}{l}\text { PTTH } \\
\text { allele }\end{array}$ & $\begin{array}{l}\text { yolk } \\
\text { allele }\end{array}$ & Fec & Hat\% & WTG & VLD(h) & TLD(h) & $\overline{S C W}(g)$ & SSW(g) \\
\hline $\begin{array}{l}\text { BBE- } \\
0001\end{array}$ & Alps jaunne & $\overline{a b}$ & aa & 368 & 91.5 & 34 & 144 & 574 & 1.53 & 0.24 \\
\hline $\begin{array}{l}\text { BBE- } \\
0002\end{array}$ & Alps Yellow & $\mathrm{aa}$ & $\overline{a b}$ & 436 & 91.4 & 34.9 & 172 & 602 & 1.63 & 0.28 \\
\hline $\begin{array}{l}\text { BBE- } \\
0003\end{array}$ & Cevenese Yellow & $\mathrm{ab}$ & $a b$ & 422 & 91.2 & 36.3 & 154 & 574 & 1.72 & 0.28 \\
\hline $\begin{array}{l}\text { BBE- } \\
0004\end{array}$ & Ascoli Yellow & $\overline{a b}$ & aa & 410 & 94.6 & 35.7 & 148 & 582 & 1.56 & 0.25 \\
\hline $\begin{array}{l}\text { BBE- } \\
0005\end{array}$ & Meigitsu & aa & $\overline{a b}$ & 403 & 92.5 & 41.9 & 147 & 577 & 1.77 & 0.32 \\
\hline $\begin{array}{l}\text { BBEI- } \\
0006\end{array}$ & B-36 & $\mathrm{ab}$ & aa & 486 & 88.2 & 41.3 & 170 & 597 & 1.73 & 0.31 \\
\hline $\begin{array}{l}\text { BBE- } \\
0007\end{array}$ & B-37 & aa & aa & 450 & 82.6 & 36.8 & 156 & 612 & 1.56 & 0.28 \\
\hline $\begin{array}{l}\text { BBE- } \\
0008\end{array}$ & B-40 & $\mathrm{ac}$ & aа & 425 & 89.4 & 35.7 & 144 & 578 & 1.55 & 0.26 \\
\hline $\begin{array}{l}\text { BBE- } \\
0009\end{array}$ & B-40 & $a b$ & $\mathrm{aa}$ & 466 & 93.1 & 41.9 & 149 & 582 & 1.56 & 0.25 \\
\hline $\begin{array}{l}\text { BBE- } \\
0010\end{array}$ & $\mathrm{~J}-112$ & aa & $\overline{a b}$ & 467 & 96.2 & 41.3 & 142 & 568 & 1.66 & 0.28 \\
\hline $\begin{array}{l}\text { BBE- } \\
0011\end{array}$ & $\mathrm{~J} 122$ & $\mathrm{ab}$ & $\overline{a b}$ & 418 & 82.2 & 36.8 & 154 & 582 & 1.91 & 0.36 \\
\hline $\begin{array}{l}\text { BBE- } \\
0012\end{array}$ & Yakwei & $\mathrm{ab}$ & aa & 460 & 92.2 & 37.9 & 159 & 592 & 1.56 & 0.28 \\
\hline $\begin{array}{l}\text { BBE- } \\
0013\end{array}$ & Chaung Naung & $\mathrm{aa}$ & $\mathrm{aa}$ & 453 & 96.5 & 36 & 146 & 576 & 1.6 & 0.29 \\
\hline $\begin{array}{l}\text { BBE- } \\
0014\end{array}$ & C-122 & aa & aа & 467 & 93.8 & 40.3 & 154 & 582 & 1.64 & 0.28 \\
\hline $\begin{array}{l}\text { BBE- } \\
0015\end{array}$ & $\begin{array}{ll}\text { C-108 } \\
\end{array}$ & $\mathrm{aa}$ & aa & 394 & 93.4 & 46.6 & 155 & 584 & 1.54 & 0.26 \\
\hline $\begin{array}{l}\text { BBEI- } \\
0016\end{array}$ & C-110 & $\mathrm{aa}$ & $\mathrm{aa}$ & 449 & 96.8 & 35.6 & 158 & 588 & 1.69 & 0.3 \\
\hline $\begin{array}{l}\text { BBE- } \\
0017\end{array}$ & Chukwei & $\overline{a b}$ & aa & 458 & 90.7 & 40 & 145 & 572 & 1.57 & 0.27 \\
\hline $\begin{array}{l}\text { BBE- } \\
0018\end{array}$ & Chinese Farmer & $a b$ & $\overline{b b}$ & 402 & 90.8 & 38.4 & 161 & 590 & 1.54 & 0.26 \\
\hline $\begin{array}{l}\text { BBE- } \\
0019\end{array}$ & $\begin{array}{l}\text { Chinese Golden- } \\
70\end{array}$ & $\mathrm{ab}$ & $\mathrm{bb}$ & 440 & 87.2 & 38.1 & 142 & 572 & 1.53 & 0.24 \\
\hline $\begin{array}{l}\text { BBE- } \\
0020\end{array}$ & $\begin{array}{l}\text { Chinese Golden- } \\
80\end{array}$ & $\mathrm{aa}$ & $\overline{\mathrm{bb}}$ & 408 & 94.3 & 36.1 & 177 & 630 & 1.34 & 0.22 \\
\hline
\end{tabular}




\begin{tabular}{|c|c|c|c|c|c|c|c|c|c|c|}
\hline $\begin{array}{l}\text { BBE- } \\
0021\end{array}$ & $\begin{array}{l}\text { Chinese Golden } \\
-90\end{array}$ & ac & $\mathrm{bb}$ & 425 & 92.8 & 27.7 & 138 & 573 & 1.67 & 0.28 \\
\hline $\begin{array}{l}\text { BBE- } \\
0022\end{array}$ & Haulak & $\mathrm{ac}$ & $\mathrm{bb}$ & 427 & 78.4 & 41.8 & 148 & 582 & 1.46 & 0.26 \\
\hline $\begin{array}{l}\text { BBE- } \\
0023\end{array}$ & King Haung & ac & $\mathrm{bb}$ & 376 & 89.9 & 35 & 162 & 592 & 1.65 & 0.28 \\
\hline $\begin{array}{l}\text { BBEI- } \\
0024\end{array}$ & Hauchi & $\mathrm{ac}$ & $\mathrm{ab}$ & 458 & 94.2 & 40.5 & 152 & 588 & 1.79 & 0.31 \\
\hline $\begin{array}{l}\text { BBE- } \\
0025\end{array}$ & Nan Naung 6A & $\mathrm{ac}$ & $\mathrm{ab}$ & 375 & 88.9 & 38.6 & 148 & 571 & 1.61 & 0.38 \\
\hline $\begin{array}{l}\text { BBE- } \\
0026\end{array}$ & Nan Naung 6D & $\mathrm{bc}$ & $\mathrm{ab}$ & 382 & 88.1 & 32 & 142 & 577 & 1.39 & 0.24 \\
\hline $\begin{array}{l}\text { BBE- } \\
0027\end{array}$ & Chinese Yellow & bc & aa & 365 & 93.8 & 37.5 & 152 & 575 & 1.72 & 0.28 \\
\hline $\begin{array}{l}\text { BBE- } \\
0028\end{array}$ & Azad & $\mathrm{ab}$ & аa & 419 & 91.4 & 38 & 152 & 578 & 1.58 & 0.26 \\
\hline $\begin{array}{l}\text { BBE- } \\
0029\end{array}$ & Azerbaijan & $a b$ & $\mathrm{ab}$ & 383 & 97 & 37.5 & 160 & 592 & 1.61 & 0.27 \\
\hline $\begin{array}{l}\text { BBE- } \\
0030\end{array}$ & Sanish E1(P) & $\mathrm{ab}$ & $\mathrm{ab}$ & 395 & 91.1 & 33.9 & 160 & 604 & 1.47 & 0.27 \\
\hline $\begin{array}{l}\text { BBE- } \\
0031\end{array}$ & Sanish-E1(M) & $a b$ & $a b$ & 412 & 95.2 & 39.4 & 163 & 600 & 1.7 & 0.28 \\
\hline $\begin{array}{l}\text { BBE- } \\
0032\end{array}$ & Sanish-E2(M) & $\mathrm{ac}$ & $\mathrm{ab}$ & 529 & 95.1 & 38.5 & 156 & 587 & 1.7 & 0.32 \\
\hline $\begin{array}{l}\text { BBE- } \\
0033\end{array}$ & Sanish-8 & ac & aа & 464 & 91.8 & 34.5 & 172 & 610 & 1.47 & 0.27 \\
\hline $\begin{array}{l}\text { BBE- } \\
0034\end{array}$ & Sanish-17 & ac & $\mathrm{ab}$ & 455 & 90.2 & 32.8 & 158 & 604 & 1.47 & 0.25 \\
\hline $\begin{array}{l}\text { BBE- } \\
0035\end{array}$ & . Sanish-18(M) & $\mathrm{bc}$ & aa & 390 & 91.4 & 39.1 & 162 & 596 & 1.63 & 0.29 \\
\hline $\begin{array}{l}\text { BBE- } \\
0036\end{array}$ & Sanish-18 (P) & $\mathrm{ac}$ & aa & 495 & 94.3 & 37.7 & 168 & 601 & 1.57 & 0.3 \\
\hline $\begin{array}{l}\text { BBE- } \\
0037\end{array}$ & Sanish-30 & $\mathrm{ac}$ & aа & 445 & 95.7 & 39.1 & 146 & 579 & 1.61 & 0.28 \\
\hline $\begin{array}{l}\text { BBE- } \\
0038\end{array}$ & . Sanish-21 & $\mathrm{ac}$ & $a b$ & 482 & 92.6 & 35.8 & 156 & 590 & 1.7 & 0.29 \\
\hline $\begin{array}{l}\text { BBE- } \\
0039\end{array}$ & Shekhi-1 & $\mathrm{ac}$ & $\mathrm{ab}$ & 485 & 89.5 & 37.8 & 152 & 584 & 1.56 & 0.28 \\
\hline $\begin{array}{l}\text { BBE- } \\
0040\end{array}$ & Sheikhi-11 & ac & aа & 450 & 86.5 & 39.2 & 167 & 598 & 1.71 & 0.32 \\
\hline $\begin{array}{l}\text { BBE- } \\
0041\end{array}$ & Tashkahashi-112 & ac & aа & 448 & 89.8 & 39.1 & 162 & 596 & 1.64 & 0.29 \\
\hline
\end{tabular}




\begin{tabular}{|c|c|c|c|c|c|c|c|c|c|c|}
\hline $\begin{array}{l}\text { BBE- } \\
0042\end{array}$ & Gyandza & aа & aа & 516 & 87.3 & 35.8 & 162 & 594 & 1.58 & 0.28 \\
\hline $\begin{array}{l}\text { BBE- } \\
0043\end{array}$ & Belkokona-11 & $\mathrm{ac}$ & aа & 485 & 93.5 & 37.8 & 168 & 604 & 1.72 & 0.29 \\
\hline BBI-0044 & NB4D2 & ac & aа & 479 & 92.1 & 34.4 & 206 & 646 & 1.82 & 0.36 \\
\hline BBI-0045 & SH-6 & $\mathrm{ac}$ & aа & 518 & 95.3 & 34.8 & 172 & 612 & 1.46 & 0.26 \\
\hline BBI-0046 & YS-3 & aа & $\mathrm{bb}$ & 438 & 94.4 & 37.8 & 156 & 598 & 1.5 & 0.27 \\
\hline BBI-0047 & SF-19 & ac & aa & 504 & 96 & 34.4 & 180 & 623 & 1.41 & 0.27 \\
\hline BBI-0048 & JD6 & ac & aа & 475 & 95.4 & 33.9 & 164 & 596 & 1.53 & 0.27 \\
\hline $\begin{array}{l}\text { BBE- } \\
0049\end{array}$ & UKR-1 & $\mathrm{ac}$ & $\mathrm{bb}$ & 499 & 87.6 & 32.9 & 190 & 628 & 1.67 & 0.3 \\
\hline $\begin{array}{l}\text { BBE- } \\
0050\end{array}$ & UKR-2 & $\mathrm{CC}$ & $\mathrm{ab}$ & 483 & 91.6 & 36.4 & 185 & 621 & 1.79 & 0.36 \\
\hline $\begin{array}{l}\text { BBE- } \\
0051\end{array}$ & Merefa-6 & aа & aа & 376 & 94 & 35.8 & 158 & 596 & 1.63 & 0.33 \\
\hline BBI-0052 & Jam-1 & ac & aа & 421 & 84.1 & 34.2 & 172 & 606 & 1.6 & 0.23 \\
\hline BBI-0053 & Jam-2 & $\mathrm{ac}$ & aа & 426 & 86 & 32.5 & 166 & 596 & 1.56 & 0.25 \\
\hline BBI-0054 & Jam-11 & $\mathrm{ac}$ & aa & 360 & 85.8 & 33.6 & 158 & 590 & 1.46 & 0.24 \\
\hline BBI-0055 & Jam-121 & $\mathrm{bc}$ & $\mathrm{ab}$ & 432 & 88.2 & 38.4 & 186 & 616 & 1.71 & 0.35 \\
\hline BBI-0056 & Jam-21 & $\mathrm{ac}$ & aa & 407 & 89 & 30.9 & 160 & 606 & 1.55 & 0.27 \\
\hline BBI-0057 & Jam-23 & ac & $\mathrm{ab}$ & 439 & 84.5 & 33.7 & 158 & 594 & 1.7 & 0.28 \\
\hline BBI-0058 & . Jam-27 & $\mathrm{ac}$ & aa & 435 & 88.5 & 36.4 & 163 & 597 & 1.54 & 0.26 \\
\hline BBI-0059 & Jam-103 & aа & $\mathrm{bb}$ & 417 & 92.6 & 38.1 & 152 & 586 & 1.53 & 0.26 \\
\hline BBI-0060 & Jam-110 & aa & aа & 392 & 92 & 38.7 & 161 & 596 & 1.49 & 0.24 \\
\hline BBI-0061 & Jam-118 & ac & aa & 430 & 82.4 & 30.6 & 159 & 588 & 1.75 & 0.31 \\
\hline BBI-0062 & Jam-119 & aa & aa & 514 & 91.5 & 35.2 & 164 & 596 & 1.74 & 0.3 \\
\hline BBI-0063 & Jam-122 & $\mathrm{ac}$ & $\mathrm{ab}$ & 411 & 89.7 & 30.6 & 206 & 648 & 1.54 & 0.27 \\
\hline BBI-0064 & Jam-124 & $\mathrm{ac}$ & aа & 385 & 89.7 & 35.6 & 181 & 616 & 1.54 & 0.27 \\
\hline BBI-0065 & Jam-125 & aa & $\mathrm{ab}$ & 433 & 95.8 & 34.2 & 166 & 604 & 1.69 & 0.3 \\
\hline BBI-0066 & Pam-101 & aa & $a b$ & 467 & 96.3 & 34.4 & 168 & 604 & 1.58 & 0.27 \\
\hline BBI-0067 & Pam-102 & $\mathrm{ac}$ & aa & 508 & 89.3 & 36.3 & 145 & 577 & 1.65 & 0.25 \\
\hline BBI-0068 & Pam-103 & $\mathrm{aa}$ & aа & 409 & 82.4 & 36.4 & 144 & 574 & 1.58 & 0.28 \\
\hline BBI-0069 & Pam-104 & aa & aa & 430 & 92.5 & 35.9 & 168 & 598 & 1.62 & 0.28 \\
\hline BBI-0070 & Pam-105 & aа & aа & 438 & 90.2 & 33.6 & 174 & 611 & 1.65 & 0.31 \\
\hline BBI-0071 & Pam-106 & $a b$ & aа & 430 & 88.2 & 37 & 169 & 602 & 1.6 & 0.29 \\
\hline
\end{tabular}




\begin{tabular}{|l|l|l|l|l|r|r|r|r|r|r|} 
& & & & & & & & & & \\
\hline BBI-0072 & Pam-107 & ac & aa & 451 & 86.8 & 39.7 & 156 & 590 & 1.66 & 0.3 \\
\hline BBI-0073 & Pam-108 & ac & aa & 392 & 85.8 & 38.4 & 189 & 610 & 1.54 & 0.29 \\
\hline BBI-0074 & Pam-109 & ac & aa & 413 & 84.1 & 36.6 & 176 & 608 & 1.68 & 0.32 \\
\hline BBI-0075 & Pam-110 & ac & aa & 412 & 88.1 & 35.1 & 162 & 594 & 1.58 & 0.3 \\
\hline
\end{tabular}

Table. 2. Allelic variations in PTTH \& yolk protein gene primers in multivoltine accessions 


\begin{tabular}{|c|c|c|c|c|c|c|c|c|c|c|}
\hline ACC.NO & Races & $\begin{array}{l}\text { PTTH } \\
\text { allele }\end{array}$ & $\begin{array}{l}\text { yolk } \\
\text { allele }\end{array}$ & Fecundity & Hat\% & WTG & VLD(h) & TLD(h) & $\mathrm{SCW}(\mathrm{g})$ & SSW(g) \\
\hline $\begin{array}{l}\text { BMI- } \\
0001\end{array}$ & Sarupat & $\mathrm{ac}$ & $\mathrm{bc}$ & 405 & 85.7 & 25.21 & 149 & 569 & 1.16 & 0.17 \\
\hline $\begin{array}{l}\text { BMI- } \\
0003\end{array}$ & Moria & $\overline{a b}$ & $\mathrm{bc}$ & 388 & 86 & 26.85 & 145 & 564 & 1.13 & 0.16 \\
\hline $\begin{array}{l}\text { BMI- } \\
0004\end{array}$ & T.white & ac & $\mathrm{bc}$ & 429 & 86.8 & 26.41 & 147 & 566 & 1.21 & 0.19 \\
\hline BMI0008 & Kolar gold & ac & $\mathrm{bc}$ & 477 & 87.6 & 27.85 & 134 & 553 & 1.26 & 0.19 \\
\hline $\begin{array}{l}\text { BME- } \\
0012\end{array}$ & R. Daizo & ac & bc & 433 & 88.6 & 29.27 & 168 & 572 & 1.41 & 0.21 \\
\hline $\begin{array}{l}\text { BMEI- } \\
0013\end{array}$ & G.Plain & $\mathrm{ac}$ & CC & 417 & 90.3 & 24.32 & 135 & 558 & 1.13 & 0.17 \\
\hline $\begin{array}{l}\text { BMI- } \\
0015\end{array}$ & Raj & ac & $\mathrm{bb}$ & 383 & 91 & 21.06 & 166 & 578 & 1.06 & 0.15 \\
\hline $\begin{array}{l}\text { BMI- } \\
0016\end{array}$ & $\bar{G}$ & $\mathrm{ac}$ & aa & 432 & 90.4 & 25.37 & 149 & 571 & 1.22 & 0.18 \\
\hline $\begin{array}{l}\text { BMI- } \\
0017\end{array}$ & Nistari & ac & aa & 381 & 82.3 & 20.91 & 143 & 557 & 1.02 & 0.18 \\
\hline $\begin{array}{l}\text { BMI- } \\
0018\end{array}$ & $\begin{array}{l}\text { Nistari } \\
\text { (M) }\end{array}$ & $\mathrm{ac}$ & aa & 392 & 90.1 & 19.64 & 142 & 559 & 0.94 & 0.13 \\
\hline $\begin{array}{l}\text { BMI- } \\
0019\end{array}$ & Nistari (p) & aa & $\mathrm{bb}$ & 382 & 88.8 & 20.58 & 134 & 549 & 0.97 & 0.14 \\
\hline $\begin{array}{l}\text { BMI- } \\
0041\end{array}$ & Wai-4 & aa & $\mathrm{bb}$ & 381 & 85.8 & 23.53 & 136 & 555 & 1.07 & 0.15 \\
\hline $\begin{array}{l}\text { BMI- } \\
0042\end{array}$ & MY-23 & $\mathrm{aa}$ & $\mathrm{bb}$ & 457 & 91.6 & 27.99 & 141 & 559 & 1.26 & 0.18 \\
\hline $\begin{array}{l}\text { BMI- } \\
0043\end{array}$ & MW13 & aa & $\mathrm{bb}$ & 470 & 88.6 & 28.96 & 138 & 558 & 1.33 & 0.2 \\
\hline $\begin{array}{l}\text { BMI- } \\
0044\end{array}$ & MHMP(W) & aa & $\mathrm{bb}$ & 407 & 88 & 29.27 & 143 & 562 & 1.31 & 0.19 \\
\hline $\begin{array}{l}\text { BMI- } \\
0045\end{array}$ & $\operatorname{MHMP}(\mathrm{Y})$ & aa & $\mathrm{bb}$ & 479 & 90.9 & 31.5 & 151 & 568 & 1.42 & 0.22 \\
\hline $\begin{array}{l}\text { BME- } \\
0046\end{array}$ & P4D3 & aa & $\mathrm{bb}$ & 461 & 92.9 & 27.85 & 153 & 571 & 1.34 & 0.21 \\
\hline $\begin{array}{l}\text { BME- } \\
0047\end{array}$ & $\begin{array}{l}\text { NISTIDI } \\
(\mathrm{Y})\end{array}$ & aa & $\mathrm{bb}$ & 426 & 90.3 & 23.77 & 143 & 558 & 1.12 & 0.15 \\
\hline $\begin{array}{l}\text { BME- } \\
0048\end{array}$ & $\begin{array}{l}\text { NISTIDI } \\
(\mathrm{W})\end{array}$ & aa & $\mathrm{bb}$ & 408 & 91 & 23.07 & 141 & 557 & 1.04 & 0.14 \\
\hline $\begin{array}{l}\text { BME- } \\
0049\end{array}$ & NK4 & aa & $\mathrm{bb}$ & 321 & 87.2 & 21.88 & 151 & 566 & 1.03 & 0.14 \\
\hline BME- & Cambodg & $\mathrm{ac}$ & $\overline{a b}$ & 315 & 83.2 & 22.19 & 139 & 556 & 0.99 & 0.14 \\
\hline
\end{tabular}




\begin{tabular}{|c|c|c|c|c|c|c|c|c|c|c|}
\hline 0050 & & & & & & & & & & \\
\hline $\begin{array}{l}\text { BME- } \\
0052\end{array}$ & Daizo & $\mathrm{ac}$ & $\mathrm{ab}$ & 393 & 87.7 & 21.76 & 162 & 564 & 0.96 & 0.13 \\
\hline $\begin{array}{l}\text { BMI- } \\
0053\end{array}$ & LMP & $\mathrm{ac}$ & $\mathrm{ab}$ & 390 & 91.7 & 23.33 & 147 & 562 & 1.07 & 0.15 \\
\hline $\begin{array}{l}\text { BMI- } \\
0054\end{array}$ & $\overline{D M R}$ & $\mathrm{ac}$ & $\mathrm{ab}$ & 410 & 90.8 & 24.13 & 138 & 549 & 1.06 & 0.16 \\
\hline $\begin{array}{l}\text { BMI- } \\
0055\end{array}$ & LMO & $\mathrm{ab}$ & $\mathrm{ab}$ & 439 & 90.9 & 25.68 & 147 & 566 & 1.19 & 0.17 \\
\hline $\begin{array}{l}\text { BMI- } \\
0056\end{array}$ & MY1(SL) & $\mathrm{bc}$ & $\overline{a b}$ & 492 & 89 & 23.98 & 153 & 581 & 1.18 & 0.18 \\
\hline $\begin{array}{l}\text { BMI- } \\
0057\end{array}$ & PM(SL) & $\mathrm{ac}$ & $\mathrm{ab}$ & 477 & 91.9 & 26.61 & 144 & 563 & 1.23 & 0.18 \\
\hline $\begin{array}{l}\text { BMI- } \\
0058\end{array}$ & BL23 & $\mathrm{ac}$ & $\mathrm{bb}$ & 491 & 92.9 & 30.74 & 151 & $\overline{559}$ & 1.4 & 0.23 \\
\hline $\begin{array}{l}\text { BMI- } \\
0059\end{array}$ & B24 & $\mathrm{ac}$ & $\mathrm{ab}$ & 472 & 82.5 & 29.07 & 148 & 562 & 1.4 & 0.23 \\
\hline $\begin{array}{l}\text { BMI- } \\
0060\end{array}$ & MU303 & ac & $a b$ & 522 & 91.2 & 27.87 & 157 & 574 & 1.34 & 0.2 \\
\hline
\end{tabular}

Table3.Cluster groups of Bivoltine silkworm accessions based on EST gene primers

\begin{tabular}{|l|c|c|l|l|}
\hline Acc. Nos. & $\begin{array}{l}\text { No. } \\
\text { of } \\
\text { acc. }\end{array}$ & Group & Alleles & $\begin{array}{l}\text { Number of } \\
\text { qualified } \\
\text { parameters }\end{array}$ \\
\hline $\begin{array}{l}\text { BBE-0001, BBE-0003, BBE-0004, BBE-0006,BBE-0011, BBE- } \\
\text { 0012, BBE-0013, BBE-0014,BBE-0016, BBE-0017, BBE-0018, } \\
\text { BBE-0019, BBE-0026, BBE-0028, BBE-0029, BBE-0030,BBE- } \\
\text { 0031, BBE-0038, BBE-0041, BBE-0042,BBI-0044, BBI-0047, BBI- } \\
\text { 0048, BBE-0051, BBI-0052, BBI-0054, BBI-0058, BBI-0061, BBI- } \\
\text { 0062, BBI-0064, BBI-0065, BBI-0066, BBI-0067, BBI-0068, BBI- } \\
\text { 0069, BBI-0070 }\end{array}$ & High & aa, ab & $\begin{array}{l}\text { Fecundity, } \\
\text { Larval growth, } \\
\text { cocoon weight, } \\
\text { Shell weight } \\
\text { and shell ratio }\end{array}$ \\
\hline $\begin{array}{l}\text { BBE-0007, BBE-0008, BBE-0009, BBE-0020, BBE-0021, BBE-0023, } \\
\text { BBE-0027, BBE-0032, BBE-0033, BBE-0034, BBE-0035, BBE- }\end{array}$ & 22 & Moderate & aa, ac & $\begin{array}{l}\text { Fecundity, } \\
\text { 0036, BBE-0037, BBE-0040, BBE-0043, BBI-0053, BBI-0063, BBI- } \\
\text { 0071, BBI-0072, BBI-0073, BBI-0074, BBI-0075 }\end{array}$ \\
$\begin{array}{l}\text { cocoon weight, } \\
\text { Shell weight } \\
\text { and shell ratio }\end{array}$ \\
$\begin{array}{l}\text { BBE-0002, BBI-0005, BBE-0010, BBE-0015, BBE-0022, BBE-0024, } \\
\text { BBE-0025, BBE-0039, BBI-0045, BBI-0046, BBE-0049, BBE-0050, } \\
\text { BBI-0055, BBI-0056,BBI-0057, BBI-0059, BBI-0060 }\end{array}$ & 17 & Low & aa, ac & $\begin{array}{l}\text { Fecundity, } \\
\text { Larval growth, } \\
\text { cocoon weight, } \\
\text { shell weight } \\
\text { and shell ratio }\end{array}$ \\
\hline
\end{tabular}


Table 4.Cluster groups of Multivoltine silkworm accessions based on EST gene primers

\begin{tabular}{|c|c|c|c|c|}
\hline Acc.Nos. & $\begin{array}{l}\text { No. } \\
\text { of } \\
\text { acc. }\end{array}$ & Group & Alleles & $\begin{array}{l}\text { Number of } \\
\text { qualified } \\
\text { parameters }\end{array}$ \\
\hline $\begin{array}{lll}\text { BMI-0001, } & \text { BMI-0007, } & \text { BME-0015, } \\
\text { BMI-0041, } & \text { BMI-0042, } & \text { BMI-0043, BME-0044, } \\
\text { BMI-0045, } & \text { BME-0046, BME-0047, BME-0048, } \\
\text { BME-0049, } & \text { BMI-0061, BMI-0062, BMI-0065, } \\
\text { BMI-0074 } & & \end{array}$ & 17 & High & $\mathrm{ac}, \mathrm{bc}$ & $\begin{array}{l}\text { Fecundity, } \\
\text { Larval } \\
\text { growth, } \\
\text { cocoon } \\
\text { weight, } \\
\text { Shell } \\
\text { weight and } \\
\text { shell ratio. }\end{array}$ \\
\hline $\begin{array}{llll}\text { BMI-0016, } & \text { BMI-0018, BME-0050, } & \text { BME-0052, } \\
\text { BMI-0053, } & \text { BMI-0054, BMI-0055, BMI-005, } \\
\text { BMI-0057 } & & & \end{array}$ & 9 & Moderate & $a a, a b$ & $\begin{array}{l}\text { Fecundity, } \\
\text { Larval } \\
\text { growth, } \\
\text { cocoon } \\
\text { weight, } \\
\text { Shell } \\
\text { weight and } \\
\text { shell ratio. }\end{array}$ \\
\hline BMI-0017, BMI-0058, BMI-0059, BMI-0060 & 4 & Low & $\mathrm{ac}, \mathrm{aa}$ & $\begin{array}{l}\text { Fecundity, } \\
\text { Larval } \\
\text { growth, } \\
\text { cocoon } \\
\text { weight, } \\
\text { Shell } \\
\text { weight and } \\
\text { shell ratio. }\end{array}$ \\
\hline
\end{tabular}

\section{Figures}




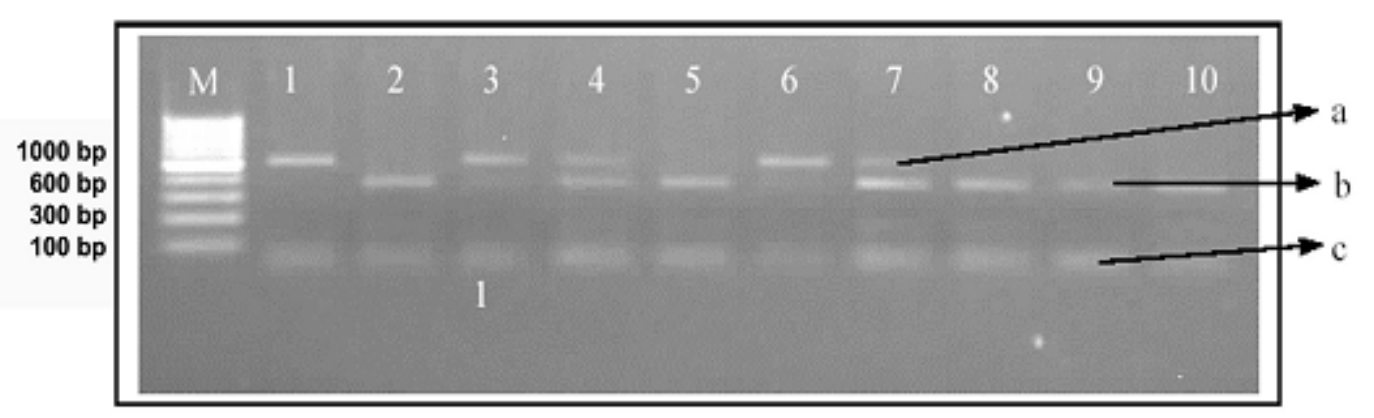

Fig.1: Restriction pattern profiles of Yolk protein gene among selected Bivoltine silkworm races

\section{Figure 1}

Restriction pattern profiles of Yolk protein gene among selected Bivoltine silworm races

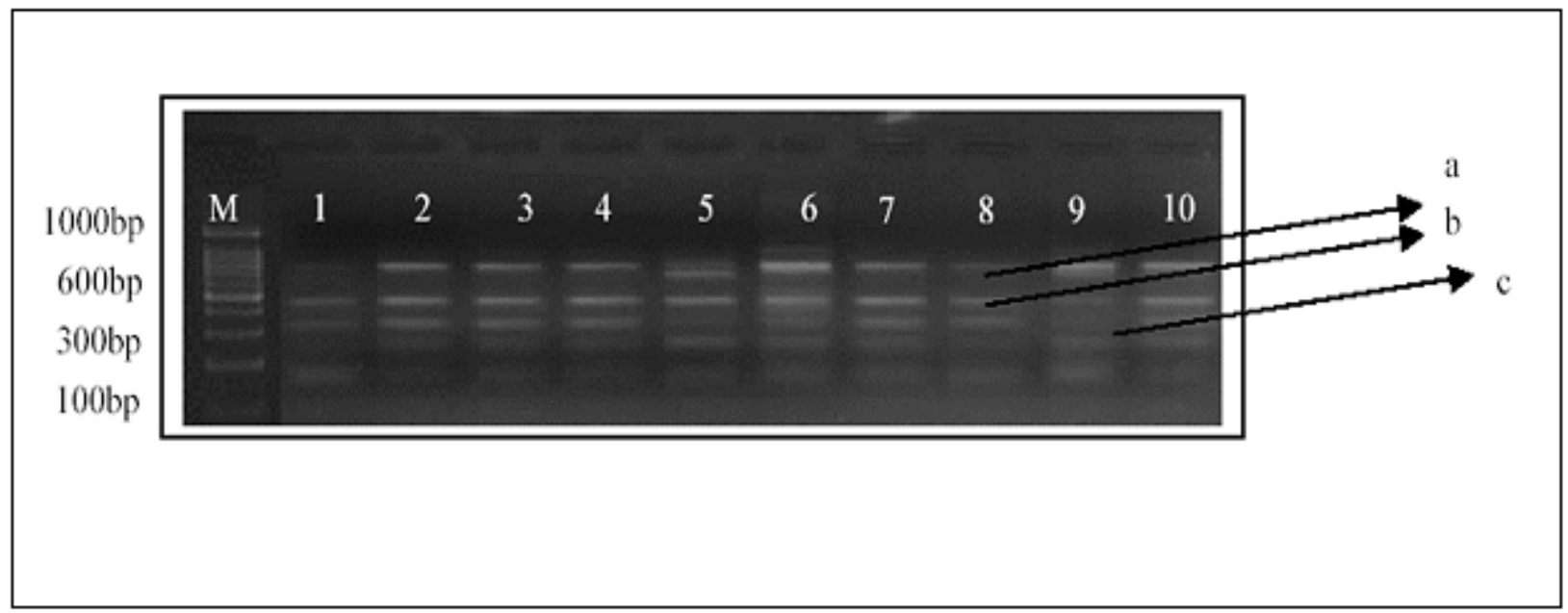

Fig; 2 Restriction pattern profiles of PTTH gene among selected Bivoltine silkworm races

Figure 2

Restriction pattern profiles of PTTH gene among selected Bivoltine silworm races 


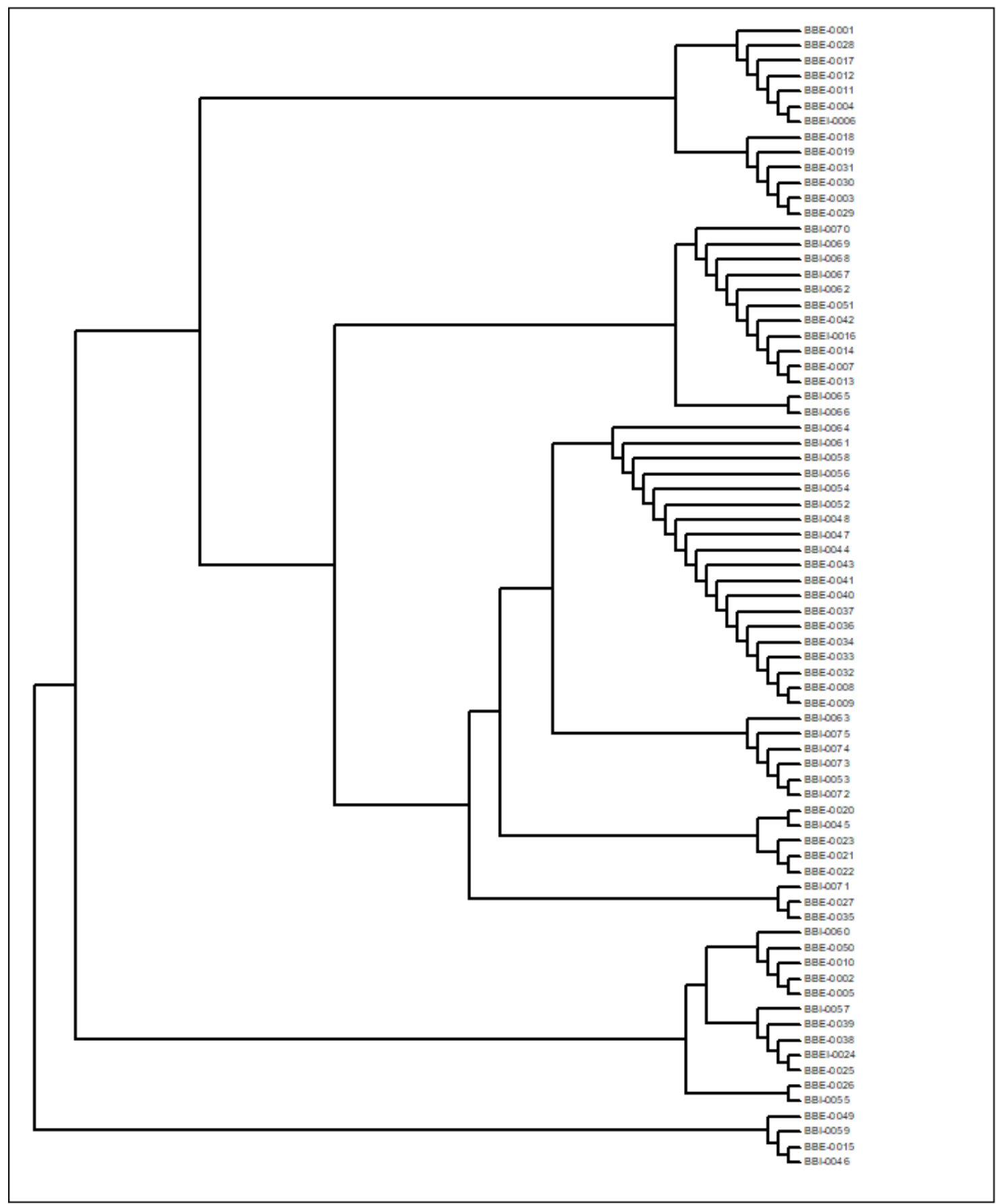

Fig 3. Dendrogram - BV based on EST gene primers

Figure 3

Dendrogram - BV based on EST gene primers 


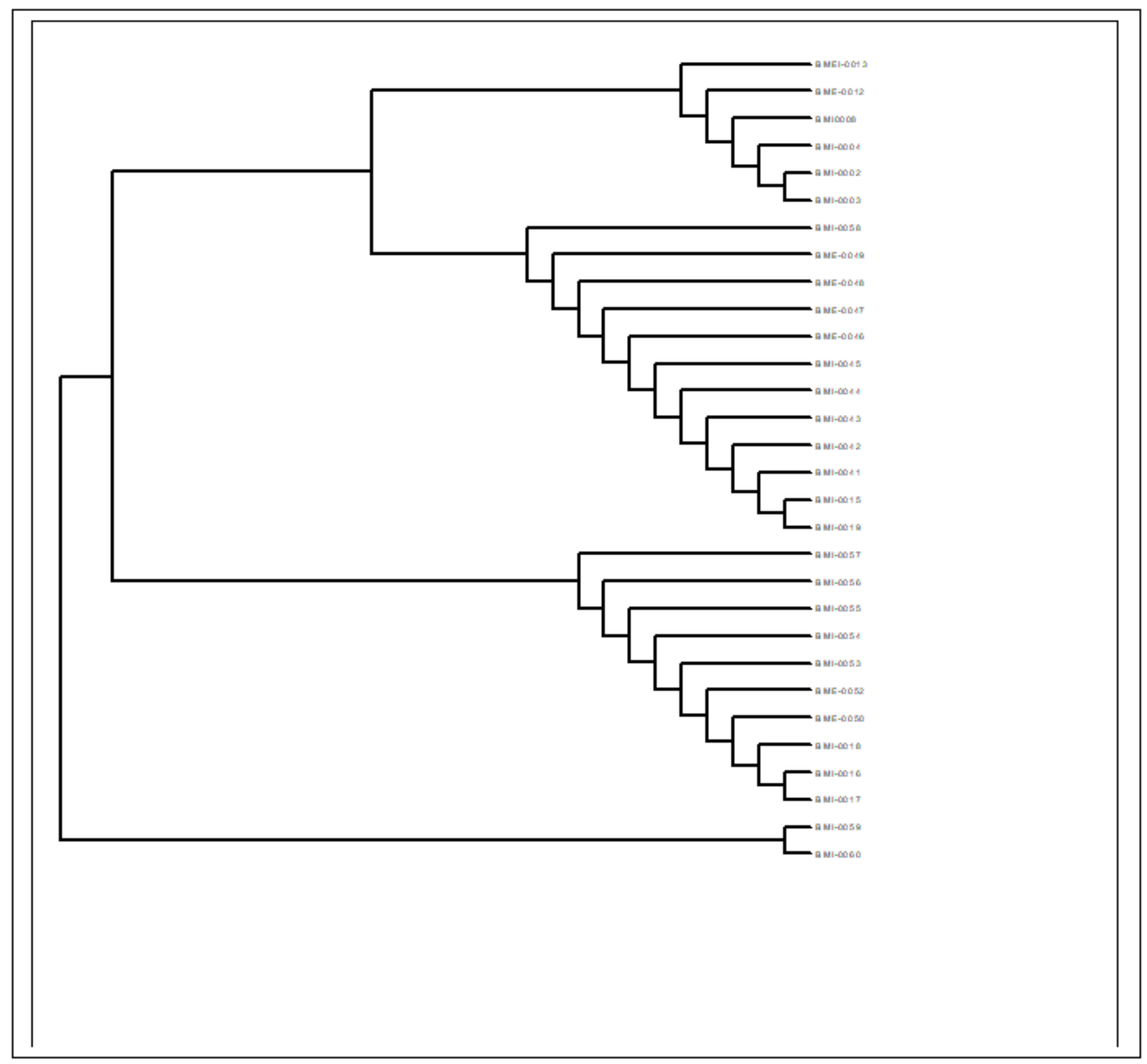

Fig 4. Dendrogram - MV based on EST gene primers

Figure 4

Dendrogram - MV based on EST gene primers 